\title{
Polyamines and ethylene during in vitro rooting of Prunus avium L.
}

\author{
S. Biondi ${ }^{1}$ and N. Bagni2 \\ ${ }^{1}$ Azienda Regionale Foreste dell'Emilia-Romagna, Bologna, and \\ 2 Dipartimento di Biologia E.S., Università di Bologna, Bologna, Italy
}

\section{Introduction}

Polyamines are widely occurring organic polycations now recognized as plant growth substances. They appear to be involved in cell division, to delay senescense and usually accompany active growth and metabolism (Bagni et al., 1982). Although a requirement for polyamines has been postulated in some in vitro morphogenetic processes, a full understanding of their role in organogenesis has yet to come. The interaction between ethylene and auxin is a wellknown phenomenon. Our primary interest in studying the action of ethylene in adventitious root formation stems from auxin usually being the key factor in root induction. Secondly, since ethylene and polyamines share a common precursor (S-adenosylmethionine) in their biosynthetic pathways, there is some evidence for a possible interrelationship between them. The aim of the present work was to begin examining the changes in the endogenous content of the polyamines, putrescine (PUT), spermidine (SPD) and spermine (SPM) and in the ethylene production accompanying the in vitro rooting of
Prunus avium shoots. In addition, the effects of externally supplied polyamines, in particular SPM, of the ethylene precursor 1-amirocyclopropane-1-carboxylic acid (ACC) and of some inhibitors of polyamine and ethylene biosyntheses, namely $\alpha$-difluoromethylornithine (DFMO), $\alpha$-difluoromethylarginine (DFMA), dicyclohexylamine (DCHA), methylglyoxal-bis-guanylhydrazone (MGBG) and aminoethoxyvinylglycine (AVG) and aminooxyacetic acid (AOA) were examined.

\section{Materials and Methods}

The experimental material is part of a routine micropropagation program for selected clones of $P$. avium L. The establishment of bud cultures and the shoot multiplication and elongation phases have been described elsewhere (De Paoli and Rocchi, 1984). Rooting experiments were carried out by transferring elongated shoots onto agar-solidified medium composed of half-strength macro- and micronutrients of Murashige and Skoog (1962) (MS), MS vitamins, $30 \mathrm{~g} /$ sucrose, $\mathrm{pH}$ 5.9-6.0, prior to autoclaving. Indole butyric acid (IBA) or indole acetic acid (IAA) was supplied at various concentrations $(2.5,5,10,25,50 \mu \mathrm{M})$ to induce 
rooting. Spermine, DFMO, DFMA, MGBG, AVG, $A O A$ and $A C C$ were filter-sterilized and added to the autoclaved medium. Cultures were kept in a growth chamber with a $16 \mathrm{~h}$ photoperiod at $22 \pm 2^{\circ} \mathrm{C}$. Polyamine levels were individually monitored at intervals in the shoot apical and basal $3 \mathrm{~mm}$ portions, stem and leaves. Dansylated derivatives of the polyamines were separated by thin-layer chromatography and fluorometrically determined. The incorporation of labeled PUT, the precursor of SPD and SPM, was evaluated by adding $185 \mathrm{kBq}$ to $100 \mathrm{ml}$ of medium (15 shoots). Shoot portions were extracted on $d 2,7$ and 15 and polyamines were analyzed as described above. Radioactivity was measured in spots comigrating with SPD and SPM. A system was developed to measure 3,4 $\left[{ }^{14} \mathrm{C}\right.$ ]methionine $(74 \mathrm{kBq} / 20 \mathrm{ml}$ of medium) incorporation into ethylene. Ten shoots were placed in small flasks equipped with a side-arm, into which filter paper soaked in $\mathrm{KOH}$ was inserted, and a center well containing either 0.1 $\mathrm{M}$ mercuric acetate (in methanol) or $0.25 \mathrm{M}$ mercuric perchlorate in order to capture the labeled ethylene formed. The radioactivity in the ethylene traps was measured after a $24 \mathrm{~h}$ incubation under normal culture conditions. The time course of methionine uptake was determined by extracting the different shoot portions in 10\% trichloroacetic acid.

\section{Results}

Of the two auxins and different concentrations tested, $5 \mu \mathrm{M}$ IBA gave the best rooting percentages after $12-15 \mathrm{~d}$ and was thus used in all subsequent experiments. Endogenous polyamine content during the rooting phase was characterized by a peak in PUT levels on d 9-11 in all shoot portions. Spermidine levels did not change significantly in leaves but showed maximum accumulation on $\mathrm{d} 9$ or 11 in other shoot portions. Spermine was always absent or present in traces. Although no labeled PUT incorporation into SPM was observed, it is worth noting the sharp peak in SPD synthesis observed in the basal portion of shoots on d 7 . Exogenously supplied SPM $(10,50$ or $100 \mu \mathrm{M})$, either in the presence of optimal or suboptimal IBA levels, had no significant effect on rooting. DCHA plus MGBG (0.5 $\mathrm{mM}$ each) markedly reduced rooting but this inhibition was only partially reversed by the simultaneous application of $0.5 \mathrm{mM}$ SPD. At higher concentration, the drugs provoked visible toxicity symptoms. DFMO plus DFMA (1 $\mathrm{mM}$ each) drastically reduced rooting as well and $0.2 \mathrm{mM}$ PUT again partially reversed this effect. As expected, treatment with these specific inhibitors of PUT biosynthesis caused a severe decline in endogenous PUT levels. DCHA plus MGBG, however, caused only a minor reduction in SPD content. ACC was supplied only at $1 \mathrm{mM}$ concentration, which proved to be lethal and thus no rooting was observed. Finally, AVG (25 or $50 \mu \mathrm{M})$ did not affect rooting percentages but enhanced the number of roots formed per rooted shoot. Results showed that mercuric perchlorate is a more efficient and reliable ethylene trap than mercuric acetate. The latter, being dissolved in methanol, quickly evaporated and, due to the presence of water vapor in the flasks, formed a yellow precipitate which was difficult to collect. The time course of labeled methionine uptake indicated that the compound was rapidly taken up (within 30 $\mathrm{min}$ ) and reached a plateau around $15 \mathrm{~h}$ in all shoot portions. Preliminary data concerning ethylene biosynthesis indicate that, upon transfer to a rooting medium containing $5 \mu \mathrm{M}$ IBA, ethylene production was of the order of $2.2 \mathrm{pmol} / \mathrm{shoot} / \mathrm{h}$. Finally, $0.5 \mathrm{mM}$ AOA was shown to significantly reduce ethylene biosynthesis and, to a lesser extent, so did a $48 \mathrm{~h}$ exposure to DCHA plus MGBG.

\section{Discussion}

The results outlined above suggest that polyamines may be involved in the rooting 
process, probably in the stages of active cell division. In fact, increases in intracellular PUT and SPD levels preceded root protrusion and may have coincided with maximum primordium development. Also, a peak in SPD synthesis was observed on d 7 in the basal portions, which are the site of root formation. Finally, although there does not seem to be a requirement for SPM, either endogenous or exogenous, specific inhibitors of PUT and SPD biosyntheses had a clear inhibitory action on rooting. Our preliminary data seem to indicate that DCHA plus MGBG do not enhance ethylene production; this may be due to the fact that these drugs were ineffective in blocking polyamine synthesis or may suggest that the 2 biosynthetic pathways are not competitive.
Further work on the role of ACC, AVG and ethylene production in adventitious root formation is in progress.

\section{References}

Bagni N., Serafini-Fracassini D. \& Torrigiani P. (1982) Polyamines and cellular growth processes in higher plants. In: Plant Growth Substances 1982. (Wareing P.F., ed.), Academic Press, New York, pp. 473-482

De Paoli G. \& Rocchi M. (1984) Propagazione in vitro del ciliegio da legno. In: Propagazione in vitro: ricerche su alcune specie forestali. A.R.F.E.R., Bologna, pp. 100-102

Murashige T. \& Skoog F. (1962) A revised medium for rapid growth and bioassays with tobacco cultures. Physiol. Plant. 15, 473-497 